\title{
Localizing the Neural Substrate of Reflexive Covert Orienting
}

\author{
*Valerie Higenell \\ Montreal Neurological Institute \\ McGill University \\ Joshua R. Hwang \\ University of Western Ontario
}

\author{
*Brian J. White \\ Centre for Neuroscience Studies \\ Queen's University \\ Douglas P. Munoz \\ Centre for Neuroscience Studies \\ Queen's University
}

\begin{abstract}
The capture of covert spatial attention by salient visual events influences subsequent gaze behavior. A task irrelevant stimulus (cue) can reduce (Attention capture) or prolong (Inhibition of return) saccade reaction time to a subsequent target stimulus depending on the cue-target delay. Here we investigated the mechanisms that underlie the sensory-based account of AC/IOR by manipulating the visual processing stage where the cue and target interact. In Experiment 1, liquid crystal shutter goggles were used to test whether AC/IOR occur at a monocular versus binocular processing stage (before versus after signals from both eyes converge). In Experiment 2, we tested whether visual orientation selective mechanisms are critical for AC/IOR by using oriented "Gabor" stimuli. We found that the magnitude of AC and IOR was not different between monocular and interocular viewing conditions, or between iso- and ortho-oriented cue-target interactions. The results suggest that the visual mechanisms that contribute to AC/IOR arise at an orientation-independent binocular processing stage.
\end{abstract}

Keywords: saccade, monocular, attention, orientation tuning, primary visual cortex

*Equal Contributors

\section{Introduction}

The mechanisms that underlie the control of visuospatial attention determine where we look at any moment. During everyday vision, the sudden appearance of a visual stimulus can "capture" attention (Godijn \& Theeuwes, 2002), which can initially facilitate orienting to that location, but later may inhibit orienting to that location. These reflexive, covert orienting behaviors have been termed Attention Capture (AC) and Inhibition of Return (IOR), respectively, and have been central to our understanding of visuospatial orienting in real world contexts (Fecteau and Munoz 2006; Klein 2000; Klein and MacInnes 1999; Jonides 1981; Posner et al 1985; Sumner et al. 2002).

Traditionally, a cue-target task has been used to investigate AC and IOR (Posner and Cohen 1984): an irrel- evant visual stimulus (the cue) is flashed briefly to the left or right of a fixation stimulus, and the participant is required to respond (either manually or with a saccadic eye movement) to the appearance of a visual target at the same (cued) or different (uncued) location as the preceding cue. When the time between the cue and target (CueTarget Onset Asynchrony; CTOA) is short (typically < $200 \mathrm{~ms}$ ), responses to the target at the cued location are faster than to uncued locations (AC). When the CTOA is long (typically $>200 \mathrm{~ms}$ ), responses to the target at the cued location are slower than to uncued locations (IOR) (Jonides 1981; Klein 2009; Klein 2000; Maylor, 1985; Posner \& Cohen, 1984).

The neural substrates responsible for AC and IOR are still not well understood. Behavioral studies in humans with damage to various brain areas support the superior colliculus (SC) as a likely candidate (Danziger et al 1997, Posner et al 1985, Sapir et al 1999, Sapir et al 2002, Sereno et al 2006). Also, direct neural correlates of 
AC and IOR have been identified in the SC of monkeys trained to perform the cue-target task (Bell et al. 2004; Dorris et al. 2002; Fecteau et al. 2004; Fecteau and Munoz 2005). In these studies, neurons in the intermediate SC layers ( $\mathrm{SCi}$ ) show an enhanced target related visual response following a cue at the same location at a short CTOA (typically $<200 \mathrm{~ms}$ ). The magnitude of this enhanced target related response is correlated with the faster reaction times associated with AC. This sensory based neural correlate of AC was only observed in the $\mathrm{SCi}$, and not the superficial SC layers (SCs). Because the SCs receives visual inputs relatively early in the visual processing stream (retina, V1), whereas the SCi only receives visual inputs from brain areas relatively late in the visual processing stream (i.e., frontal, parietal, and extrastriate visual cortices; for a recent review of the SC see White and Munoz, 2011), the neural substrates responsible for AC have been proposed to arise from these later areas, or within the SCi itself (Fecteau and Munoz 2005).

In contrast, there is evidence of a neural correlate of IOR in both the SCs and the SCi. In this case, neurons in both regions show an attenuated target related visual response when followed by a cue at the same location at a long CTOA (typically > 200ms; Bell et al. 2004, Dorris et al. 2002; Fecteau et al. 2004; Fecteau and Munoz 2005). The magnitude of this attenuated target related response is strongly correlated with the slower reaction times associated with IOR.

However, it is not clear whether the SC is the critical substrate underlying these orienting behaviors. There is evidence that the SC is not inhibited during IOR, but that the visual attenuation arises upstream of the SC neurons they recorded (Dorris et al. 2002). Because the SCs receives projections from very early stages of processing (retina, V1), and the $\mathrm{SCi}$ receives projections from relatively later stages of processing (frontal and parietal inputs) (Fries, 1984; Lock et al., 2003), the mechanism responsible for IOR likely arises early in the processing stream because a neural correlate of it is already observed in the SCs. That is, the attenuated target related response associated with IOR is unlikely to arise from parietal or frontal inputs because the earliest part of this response is already attenuated in the SCs (Bell et al. 2004; Fecteau et al. 2004; Fecteau and Munoz 2005), which does not receive inputs from these areas. This narrows the potential locus of IOR in particular.
The aim of this study is to elucidate the neural substrate(s) underlying this sensory based account of AC and IOR by manipulating the visual processing stage where the cue and target interact (Fig. 1). In Experiment 1 (Visual Occlusion Manipulation), we tested whether AC or IOR arise at a monocular processing stage (i.e., before visual signals from both eyes converge) versus a binocular processing stage (after visual signals from both eyes converge). This was achieved by using liquid crystal shutter goggles which controlled visual input to the left and right eyes independently, thereby controlling the processing stage (monocular vs. binocular) in which these signals could interact (Fig. 2B). We asked whether presenting the cue and target to different eyes (interocular viewing) produces the same pattern of $\mathrm{AC}$ and IOR as when presenting the cue and target to the same eye (monocular viewing) or both eyes (binocular viewing). If the critical interaction between cue and target that produces AC and IOR occurs at a monocular stage (i.e., retina or lateral geniculate nucleus; Monocular Hypothesis, Fig. 1 ), we predict that the cuing effects will be attenuated in the interocular viewing condition (red line, Fig. 1B), but not the monocular viewing condition (blue line, Fig. 1B). Alternatively, If the critical interaction between cue and target that produces $\mathrm{AC}$ or IOR occurs at a binocular stage (e.g., visual cortex or SC; Binocular Hypothesis, Fig. 1), the magnitude of AC and IOR should be similar between interocular and monocular/binocular conditions (Fig. 1C).

Experiment 2 (Stimulus Orientation Manipulation) was aimed at further exploring the Binocular Hypothesis, because this hypothesis comprises several possible brain areas (Fig. 1A). We exploited the fact that orientation is a predominant tuning property of neurons in primary visual cortex (V1) in particular (Ferster and Miller 2000; Hubel and Wiesel 1968, 1975), but not the SC (Cynader and Berman,1972). Also, recent evidence suggests that IOR is attenuated in monkeys with a V1 lesion (Ikeda et al., 2011). We tested whether some component of AC or IOR occurs through orientation selective mechanisms in visual cortex. This was achieved by using carefully controlled oriented "Gabor" stimuli (see Methods), such that the cue and target could be the same (iso-orientation) or different (orthogonal-orientation) orientation (Fig. 2C). We reasoned that, if $\mathrm{AC}$ and IOR involve a pathway through orientation selective mechanisms in visual cortex, the magnitude of these effects should be influenced by the degree to which the cue and target drive the same versus 
different orientation selective mechanisms. If so, we predict that $\mathrm{AC}$ and IOR will be attenuated when the cue and target are of different orientation (red line, Fig. 1B) compared to the same orientation (blue line, Fig. 1B). Figure 1B depicts the extreme case in which AC and IOR depend entirely or not at all on orientation selective mechanisms.
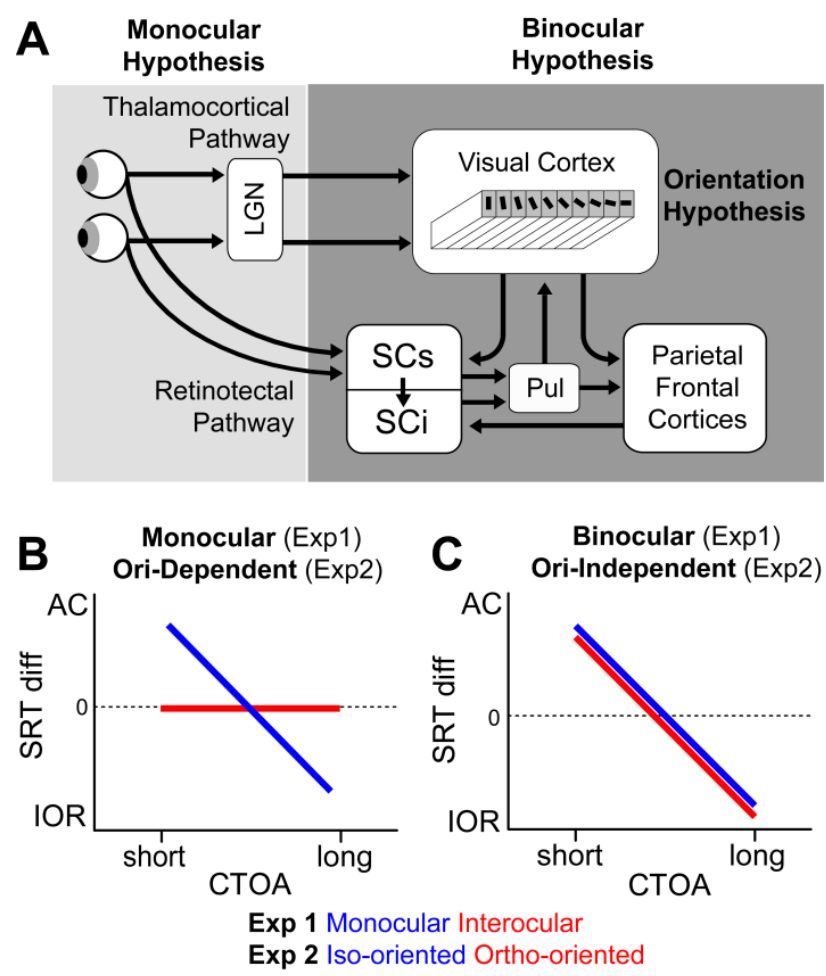

Figure 1: Schematic of visual pathways and structures implicated in our hypotheses (A). Exp. 1 tested the hypothesis that AC/IOR occur at a monocular processing stage (before visual signals from both eyes converge) versus a binocular processing stage (after visual signals from both eyes converge). Exp. 2 tested the hypothesis that AC/IOR involve a pathway through orientation selective mechanisms in visual cortex. Panels $\boldsymbol{B}$ and $\boldsymbol{C}$ show the hypothesized pattern of results produced by subtracting the saccadic reaction time (SRT diff) to a target preceded by a cue in the opposite location minus the same location. The Monocular Hypothesis predicts attenuated AC/IOR during interocular viewing $(\boldsymbol{B})$. The Binocular Hypothesis predicts equal magnitude AC/IOR between monocular and interocular viewing conditions $(\boldsymbol{C})$. The Orientation-Dependent Hypothesis predicts attenuated AC/IOR when the orientation of the cue and target are different (ortho-oriented) versus the same (isooriented) (B). The Orientation-Independent Hypothesis predicts equal magnitude AC/IOR between stimulus orientation conditions $(\boldsymbol{C})$. Abbreviations: LGN-lateral geniculate nucleus; Pul - Pulvinar; SC - superior colliculus; SCi - SC intermediate layers; SCs - SC superficial layers.

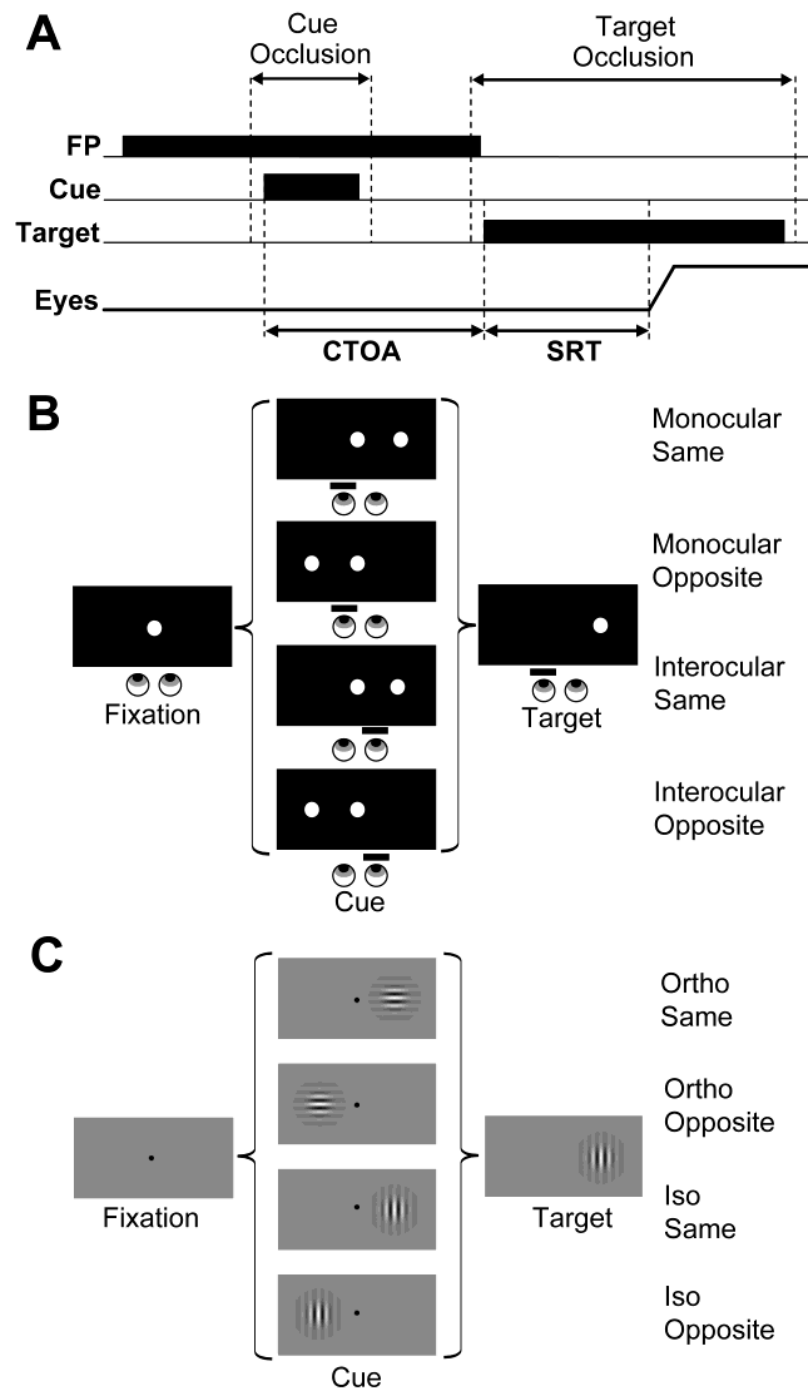

Figure 2: Schematic illustrating the series of events across trials. A. Each trial began with the subject fixating a central fixation point (FP). The cue was then flashed briefly to the left or right of fixation $\left(5^{\circ} \mathrm{vs} 10^{\circ}\right.$ eccentricity in Exp. 1 and 2, respectively). After a delay (CTOA) the target appeared at the same or opposite position as the cue. SRT to the target was measured. $\boldsymbol{B}$. Schematic of the Visual Occlusion Manipulation (Exp. 1). Prior to cue appearance, the left, right, both, or neither lens was occluded so that the cue was presented to one, neither, or both eyes. The lenses became transparent after the disappearance of the cue. Prior to target appearance, the left, right, or neither lenses were again occluded and the target was presented to the same or opposite location as the cue. $\boldsymbol{C}$. Schematic of the Stimulus Orientation Manipulation (Exp. 2). The cue and target were oriented Gabor stimuli (4 cycles per degree) presented at either the same (iso-oriented) or orthogonal (ortho-oriented) orientation. Here, the eyes were not occluded. 


\section{Methods}

\section{Participants}

The participants $(\mathrm{N}=10$ and $\mathrm{N}=18$ for Experiments 1 and 2, respectively) ranged in age from 19 to 32 years, had no known visual, psychiatric, or neurological disorders, and had normal or corrected-to-normal vision. They were remunerated for their participation. Both experiments in this study were approved by the Queen's University Human Research Ethics Board, in accordance with the Declaration of Helsinki.

\section{Visual Occlusion Manipulation (Exp. 1)}

Adjustable head-mounted liquid crystal shutter goggles with lenses that could be occluded independently were created by attaching the lenses to welder's goggles with two individual connected eyepieces preventing contamination from ambient light (model 5444T5; McMaster-Carr). The lenses (model LV4500P-OEM; Displaytech) used Ferroelectric Liquid Crystal technology to occlude vision when a voltage change was applied. Because the goggles had two separate eyepieces, each eye could be occluded individually with no consequence on the other eye.

Horizontal eye position was recorded using direct current bi-temporal electrooculography (EOG). Ag-AgCl electrodes were attached to the forehead (ground), and the outer canthus of both eyes (underneath the goggles). Using a Grass P18 amplifier, the signal from the electrodes was amplified and low-pass filtered. REX (Hays et al. 1982) was used to present the stimuli, control the shutter goggles, and record the data (see Munoz et al. 1998).

A translucent tangent screen was placed $1 \mathrm{~m}$ from the subjects' eyes and spanned $\pm 35^{\circ}$ of the visual field. A central red light-emitting diode (LED; $7.9 \mathrm{~cd} / \mathrm{m}^{2}$ ) served as the fixation point (FP), and another red LED (0.4 $\mathrm{cd} / \mathrm{m}^{2}$ ) positioned $5^{\circ}$ to the left and the right of central fixation on the horizontal meridian served as the cue and target. To prevent dark adaptation, the tangent screen was diffusely illuminated $\left(1 \mathrm{~cd} / \mathrm{m}^{2}\right)$ for a brief $500 \mathrm{~ms}$ during each inter-trial interval.

The participants were instructed to fixate the central FP for 800-1000 ms, and during this interval a cue appeared $5^{\circ}$ to the left or right of the FP (Fig. 2A). The cue remained visible for $30 \mathrm{~ms}$ and the participant was in- structed not to look to the cue. Shortly after, the target appeared either at the same or opposite location as the cue (simultaneous with FP disappearance). Participants were instructed to look to the target as soon as it appeared. The time between the onset of cue and target (Cue-Target Onset Asynchrony; CTOA) was 100 or 500 $\mathrm{ms}$. The target was visible for $800 \mathrm{~ms}$, after which the background illumination appeared. Cue and target location as well as CTOA were equally probable and randomly selected.

Just before a cue appeared, the left, right, both, or neither of the shutter goggles were occluded (Fig. 2B). After the cue and just before the target appeared, the left, right, or neither of the shutter goggles were again occluded. These patterns of visual occlusion were independent of each other and were randomly selected on a trial-bytrial basis. Both lenses were transparent during the intertrial interval, assuring that both eyes were exposed to the diffuse illumination during this period. Thus, the cue was presented to the right, left, both or neither eyes, and the target was then viewed with the same eye (monocular), the opposite eye (interocular), or both eyes (binocular) as the cue. The combination of CTOA, stimulus position, and cue/target occlusion resulted in some trial types that were not crucial for the purpose of this study, and added unnecessarily to the total trial count (the full factorial design would consist of 96 conditions). Therefore, the experiment did not include trial types where the cue was occluded from both eyes, and the target was occluded from one eye, reducing the total to 80 trial types. Specifically, there were 2 CTOAs (100/500ms), 2 cue positions (left/right), 2 target positions (left/right), and 10 occlusion conditions in which the cue or target was occluded from one eye (left cue, right cue, left targ, right targ), the cue and target were occluded from one eye (left cue/left targ, right cue/right targ, left cue/right targ, right cue/left targ), the cue was occluded from both eyes (left and right cue), or neither stimulus was occluded. The critical comparison was where the cue and target appeared monocularly versus interocularly.

Each participant completed 1600 trials in four or five experimental sessions. The trials were divided into 10 blocks of 160 trials, and each block contained two pseudo-randomized repetitions of the 80 trial types. Before each session of the experimental task, a short block of 2040 trials of the control task (see below) was administered to ensure proper visual occlusion. 
The onset of a saccade was marked as the time when eye velocity exceeded $30 \%$ s. A saccade was labeled correct if it began after the target appeared and was directed to the correct location. Saccades made in the wrong direction or before the appearance of target were marked as errors. Saccades were discarded if the reaction time was less than $90 \mathrm{~ms}$ (anticipation errors) or longer than 400 ms.

The latency from target appearance to the initiation of the saccade (saccadic reaction time; SRT) was used as the dependent measure. Mean correct SRTs were subjected to a repeated measures ANOVA. For this analysis, the viewing condition of the target with respect to the cue was considered (monocular, interocular, vs binocular) and was contrasted with CTOA (100 vs $500 \mathrm{~ms}$ ), and cuetarget relationship (same vs opposite location). An alpha of 0.05 was used as an index of significance. Pair-wise comparisons used the t-distribution and appropriate corrections were made due to multiple comparisons (Bonferroni).

\section{Visual Occlusion Control Task}

Ten participants performed a control task designed to assess whether the shutter goggles effectively blocked vision. The participants maintained gaze on a red FP for 800-1000 ms. Disappearance of the FP was followed by occlusion of the shutter goggles (the left, right, neither, or both), and appearance of the target $5^{\circ}$ to the left or right of the FP. The participant was required to generate a saccade to the target as soon as it appeared. Three types of catch trials were also included in which a target should not have been visible: no target without occlusion, no target with occlusion, and target appearance with occlusion. These catch trials were inserted to assess whether the stimuli could be seen through the occluded lenses and to test for guessing of target location in trials for which both lenses were occluded; in none did we expect participants to make a saccade. Each participant contributed a total of 1000 trials in two experimental sessions. The trials were divided into 5 blocks of 200, and each block consisted of twenty pseudo-randomized repetitions of the ten different trial types. Short breaks were provided between blocks.

Mean correct SRTs were analyzed using repeated measures analysis of variance and pair-wise t-tests. SRTs were contrasted between the different viewing conditions (monocular vs binocular) and the different locations of the target (left vs right). The number of saccades generated on the three types of catch trials was also analyzed.

When the target was visible, subjects generated saccades on $99.1 \%$ of the trials, and made very few direction errors $(0.05 \%$ of the trials; Fig. 3A). The participants generated saccades on only $1.2 \%$ of the catch trials, and the number of errors made between the three catch trial conditions (occlusion and no target, no occlusion and no target, occlusion and target) were equivalent, $\mathrm{F}(3,27)=$ 0.16, n.s (Fig. 3A). This indicates that the targets could not be seen when the lenses were occluded. Mean correct SRTs (Fig. 3B) were shorter when the target was viewed binocularly $(206 \mathrm{~ms} \pm 11)$ than when it was viewed monocularly $(238 \mathrm{~ms} \pm 12), \mathrm{t}(9)=9.90$, $\mathrm{p}<0.001$. This binocular advantage ("binocular summation") has been reported in a variety of different tasks, including flicker fusion, form recognition, acuity, and increment detection (Blake and Fox 1973; Minucci and Connors 1964; Poffenberger 1912). Comparing the monocular viewing conditions revealed no significant differences between trials in which the left $(227 \mathrm{~ms} \pm 13)$ or right ( $247 \mathrm{~ms} \pm 13)$ eye was occluded, $\mathrm{t}(9)=2.44$, n.s.
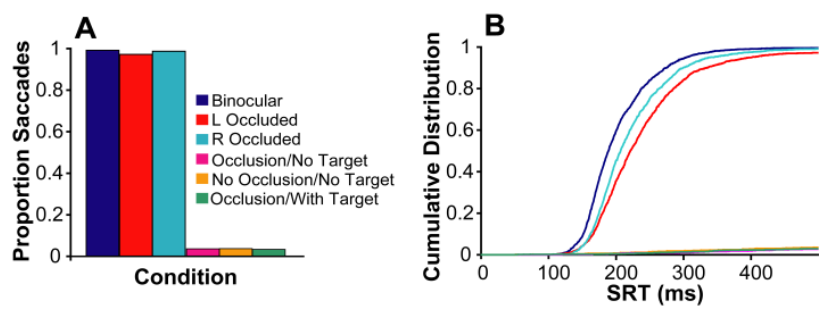

Figure 3: A. Proportion of saccades elicited as a function of condition in the Visual Occlusion Control Task. B. Cumulative SRT distribution (see Methods for details). Trial types: binocular (blue), left eye occluded (red), right eye occluded (turquoise), occlusion without target (pink), no occlusion without target (orange), and occlusion with target (green).

\section{Stimulus Orientation Manipulation (Exp. 2)}

The participant's head was stabilized with a chin rest while stimuli were presented on a Samsung SyncMaster video monitor (Model 957MB, screen resolution of 1024 x 768 pixels, refresh rate of $100 \mathrm{~Hz}$ non-interlaced, 8 bits per channel intensity resolution). Viewing distance was $50 \mathrm{~cm}$, resulting in a viewing area of $36^{\circ} \times 27^{\circ}$. The voltage to luminance relationship (gamma) was linearized using the Model S471 Portable optometer with Model 2153 filter (UDT Instruments, San Diego, CA). 
Eye position was recorded at $250 \mathrm{~Hz}$ using the Eyelink II system (SR Research, Mississauga, Ontario). Calibration was performed with a nine point stimulus routine. Custom designed software in $\mathrm{C}++$ was used to present the stimuli, and control the Eyelink. Saccades were determined from Eyelink's saccade detector, which is based on a velocity threshold of $35 \%$, and an acceleration threshold of $9500 \% \mathrm{~s}^{2}$.

Stimuli were presented on a neutral gray background at $26 \mathrm{~cd} / \mathrm{m}^{2}$ and the fixation stimulus was a black spot $0.2^{\circ}$ in diameter. Both the cue and target were stationary Gabor stimuli defined as a sine wave grating of 4 cycles ${ }^{\circ}$, windowed by a Gaussian with a standard deviation of $0.6^{\circ}$. The cue and target were vertically or horizontally oriented, and both had the same phase. They were presented at an eccentricity of $10^{\circ}$ left or right of fixation. The eyes were never occluded in this experiment.

Contrast of the target was fixed at $3 \log$ units above each participant's detection threshold in an effort to prevent a target of one orientation from being detected by mechanisms tuned to the orthogonal orientation. Detection threshold was determined separately for each participant using a 2-alternative forced choice procedure (left versus right), and an adaptive staircase procedure with a 1-up 3-down rule. This yielded the $79 \%$ correct threshold for targets at the $10^{\circ}$ left/right position (Macmillan and Creelman, 2005). Cue contrast was fixed at $50 \%$.

The participant fixated the central FP and then initiated each trial by pressing a key on a gamepad, which was followed by a random interval of 500-1000 ms before presentation of the cue (Fig. 2C). The cue was flashed for a duration of $30 \mathrm{~ms}$ to the left or right $\left(10^{\circ}\right.$ eccentricity) of the central FP. After a delay (CTOA of $100 \mathrm{~ms}$ or $500 \mathrm{~ms}$ relative to the appearance of the cue), the target was presented in either the same or opposite position as the cue, simultaneous with the removal of the FP. The participant was instructed to make a saccade to the target as soon as it appeared. SRTs less than $90 \mathrm{~ms}$ and more than $400 \mathrm{~ms}$ were removed from analysis. On one-third of the trials there was no cue. All conditions were randomly interleaved.

Each participant completed a total of 480 trials in a single experimental session (10 blocks of 48 trials each). Sessions were typically 1.5 hours and participants could take breaks between blocks if desired. All analyses were performed offline.
The latency from target appearance to the initiation of the saccade (SRT) was used as the dependent measure. Mean correct SRTs were analyzed using repeated measures analysis of variance and Bonferroni-corrected pair-wise t-tests. Analysis contrasted CTOA (100 ms vs $500 \mathrm{~ms}$ ), cue-target relationship (same vs opposite) and stimulus orientation (iso- vs ortho-oriented). An alpha of 0.05 was used as index of significance.

\section{Results}

\section{Experiment 1 (Visual Occlusion Manipulation)}

Although data were collected when there was only one occlusion per trial (e.g., an occlusion of the cue or the target but not both), only data with a cue occlusion and a target occlusion (or no occlusion for either) are reported here.

Three percent of the trials were discarded because participants looked toward the cue instead of waiting for the target to appear. Also, $0.12 \%$ of trials were discarded because participants looked in the opposite direction of the target. A main effect of occlusion was observed, $\mathrm{F}(2,18)=15.95$, $\mathrm{p}<0.001$. Mean SRT for binocular presentation $(231 \mathrm{~ms} \pm 9)$ was faster than for monocular $(242$ $\mathrm{ms} \pm 9)$ or interocular presentation $(241 \mathrm{~ms} \pm 8)$, $\mathrm{t}(9)=4.3, \mathrm{p}=0.003$, and $\mathrm{t}(9)=4.0, \mathrm{p}=0.006$, respectively (Fig. 4A; see also Fig. 3B). Participants had longer SRTs at the $100 \mathrm{~ms}$ CTOA $(267 \pm 9)$ than the $500 \mathrm{~ms}$ CTOA (209 $\mathrm{ms} \pm 9), \mathrm{F}(1,9)=212, \mathrm{p}<0.05$. This is a common finding in cueing studies, and may be related to a warning effect produced by the cue (Correa et al., 2004; Fecteau and Munoz, 2007; Tipper \& Kingstone, 2005; see also Niemi, \& Näätänen, 1981), or the fact that longer fixation durations can also lead to shorter SRTs (Paré and Munoz, 1996).

The interaction involving CTOA and cue-target relationship was significant $(\mathrm{F}(1,9)=14.36, \mathrm{p}<0.01)$, indicating the presence of a robust opposite location advantage at $500 \mathrm{~ms}$ (IOR) and a weak (non-significant) same location advantage at $100 \mathrm{~ms}$ (AC). There was no interaction between CTOA, cue-target relationship, and viewing condition $(F(1,9)<1$, n.s. $)$, indicating that the above cueing effects persisted with all types of visual occlusion. There was no difference in mean SRT for interocular or monocular stimulus presentation at the $100 \mathrm{~ms}$ CTOA 

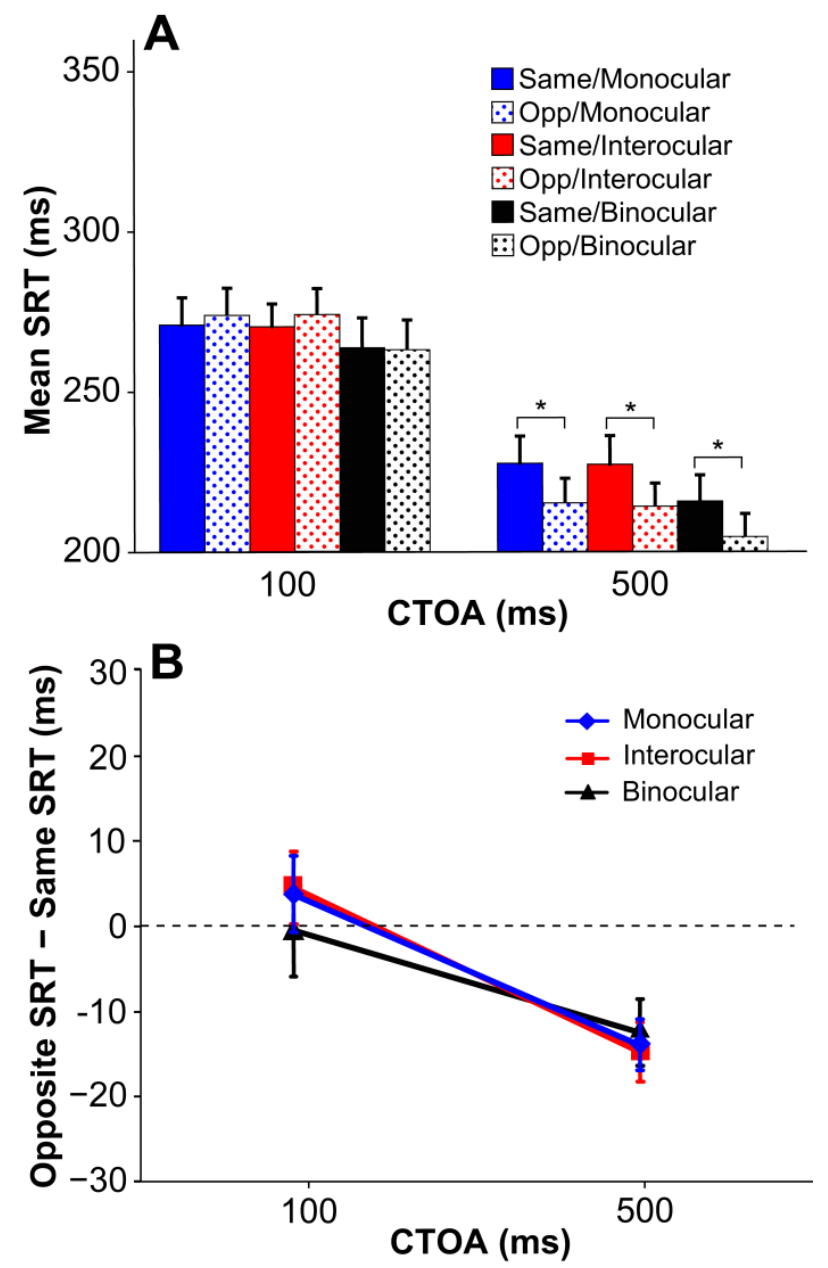

Figure 4: A. Mean SRT ( \pm 1 standard error) for same (solid bars) versus opposite (stippled bars) cue-target locations across the different goggle manipulations (Exp. 1): Monocular (blue), Interocular (red), Binocular (black). Data were collected at CTOA of $100 \mathrm{~ms}$ and $500 \mathrm{~ms}$. B. SRT differences (opposite SRT-same SRT) for Monocular, Interocular, and Binocular conditions. ${ }^{*} p<0.05$, Bonferroni corrected. There were no significant differences across goggle manipulations

$(\mathrm{t}(9)=0.15$, n.s. $)$ or at the $500 \mathrm{~ms}$ CTOA $(\mathrm{t}(9)=0.20$, n.s. $)$ (Fig. 4A).

Difference scores (opposite SRT-same SRT) were calculated and defined as AC for values greater than zero and IOR for values less than zero. The interaction between CTOA and viewing condition was not statistically significant $(\mathrm{F}(2,18)<1$, n.s. $)$. At the $500 \mathrm{~ms}$ CTOA, the difference was significantly less than zero across all viewing conditions (Fig. 4B; monocular: $t(9)=4.80$, $\mathrm{p}<0.01$, interocular: $\mathrm{t}(9)=4.37, \mathrm{p}<0.01$, binocular: $\mathrm{t}(9)=3.30, \mathrm{p}<0.01)$, indicating robust IOR. At the $100 \mathrm{~ms}$ CTOA, the differences were not significant (monocular: $\mathrm{t}(9)=0.77$, n.s., interocular: $\mathrm{t}(9)=1.0$, n.s., binocular: $\mathrm{t}(9)=$ 0.14 , n.s.), but the trend was in the direction indicative of AC. The lack of a robust effect of AC is most likely due to individual differences in optimal CTOA that produce the effect (Klein, 2000; Fecteau et al. 2005). That is, if the CTOA is not short enough, some participants begin to show a pattern indicative of IOR, instead of AC. This conclusion is consistent with the fact that three of the participants showed a pattern of moderately prolonged SRTs for cued locations at the short CTOA, indicative of IOR. If we analyze the remaining data separately, a significant main effect at both CTOAs begins to emerge. However, even with these participants removed, there was absolutely no indication of a difference between the monocular and interocular viewing conditions $(\mathrm{t}(6)=$ $0.22, \mathrm{p}=0.8$ ). Although we were not able to optimize the short CTOA for each participant to maximize the effect size, we can conclude that the magnitude of the cueing effect at the long CTOA was the same across monocular, interocular and binocular viewing conditions (Fig. 4). These results are in line with the Binocular Hypothesis (Fig. 1A), that AC and IOR arise at a stage after visual signals from both eyes converge.

\section{Experiment 2 (Stimulus Orientation Manipulation)}

The results of Experiment 1 suggest that AC and IOR arise at a processing stage after visual signals from both eyes converge (Binocular Hypothesis; Fig. 1A). However, as described earlier, there is strong evidence that the mechanism responsible for IOR in particular must arise relatively early in the visual processing stream. The neural correlate of IOR is already observed in the earliest part of the target related response in neurons from the SCs (Dorris et al. 2002; Fecteau and Munoz, 2005), which does not receive inputs from parietal or frontal cortices, but instead from the retina and primary visual cortex (White and Munoz, 2011). This limits to some degree the stage of visual processing that may contribute to it (e.g., direct retinal inputs and V1). The aim of Experiment 2 was to test the hypothesis that $\mathrm{AC}$ or IOR involve an orientation selective pathway through visual cortex.

Four percent of the trials were discarded because participants looked toward the cue instead of waiting for the target to appear. Also, $8.9 \%$ of trials were discarded because participants looked in the opposite direction of 
the target (percentage of errors per condition indicated within the bars in Fig. 5A). The greater proportion of target related direction errors was because in this experiment target contrast was intentionally reduced to $3 \log$ units above detection threshold to prevent a target of one orientation from being detected by mechanisms tuned to the orthogonal orientation (see Methods).

We ran a 3-way repeated-measures ANOVA contrasting the effect of CTOA $(100 / 500 \mathrm{~ms})$, cue-target position (same/opp) and stimulus orientation (iso/ortho) on SRT. As in Experiment 1, there was a main effect of CTOA $(F(1,17)=55, p<0.001)$. SRTs were longer at the 100ms CTOA (308 $\mathrm{ms} \pm 6)$ than at the $500 \mathrm{~ms}$ CTOA (252 $\mathrm{ms} \pm 8$ ) (Fig. 5), again likely due to the warning effect produced by the cue at the longer CTOA (Correa et al., 2004; Fecteau and Munoz, 2007; Tipper \& Kingstone, 2005; see also Niemi, \& Näätänen, 1981), or longer fixation duration which is known to produce shorter SRT (Paré and Munoz, 1996). There was also a significant interaction between CTOA and cue-target relationship, $(\mathrm{F}(1,17)=25.78, \mathrm{p}<0.01)$ (Fig. 5A), but no other interactions, including the 3 -way, were found to be statistically significant $(\mathrm{F}(1,17)<2.6, \mathrm{p}>0.13$ across remaining interactions). The 2-way interaction was due to a significant effect of $\mathrm{AC}(\mathrm{t}(17)=2.6, \mathrm{p}<0.05)$ and IOR $(\mathrm{t}(17)=4.2$, $\mathrm{p}<0.01$; one-tailed t-test on difference scores in Fig. $5 \mathrm{~B}$ collapsed across stimulus orientation). While there was no significant interaction with stimulus orientation, the main effect of orientation did reach statistically significance $(\mathrm{F}(1,17)=6.04, \mathrm{p}<0.05)$. However, post-hoc tests failed to find a significant difference between the isoversus ortho-oriented stimulus conditions $(\mathrm{t}(17)=0.36$, $\mathrm{p}=0.72$, for the $100 \mathrm{~ms}$ CTOA, and $\mathrm{t}(17)=1.6, \mathrm{p}=0.23$, for the $500 \mathrm{~ms}$ CTOA, Bonferroni corrected). Although it is tempting to argue that the main effect of orientation may be driven by a subtle difference at the 500ms CTOA (Fig $5 \mathrm{~B})$, this interpretation cannot be adequately justified without a significant post-hoc result. This is reinforced by the fact that even without a post-hoc correction the difference at the $500 \mathrm{~ms}$ CTOA was not statistically significant $(p=0.12)$. Thus, the results failed to show a reliable effect of stimulus orientation on AC or IOR. More importantly, neither effect was abolished as a function of cue-target orientation. We conclude that orientation selective mechanisms do not contribute significantly to AC or IOR.
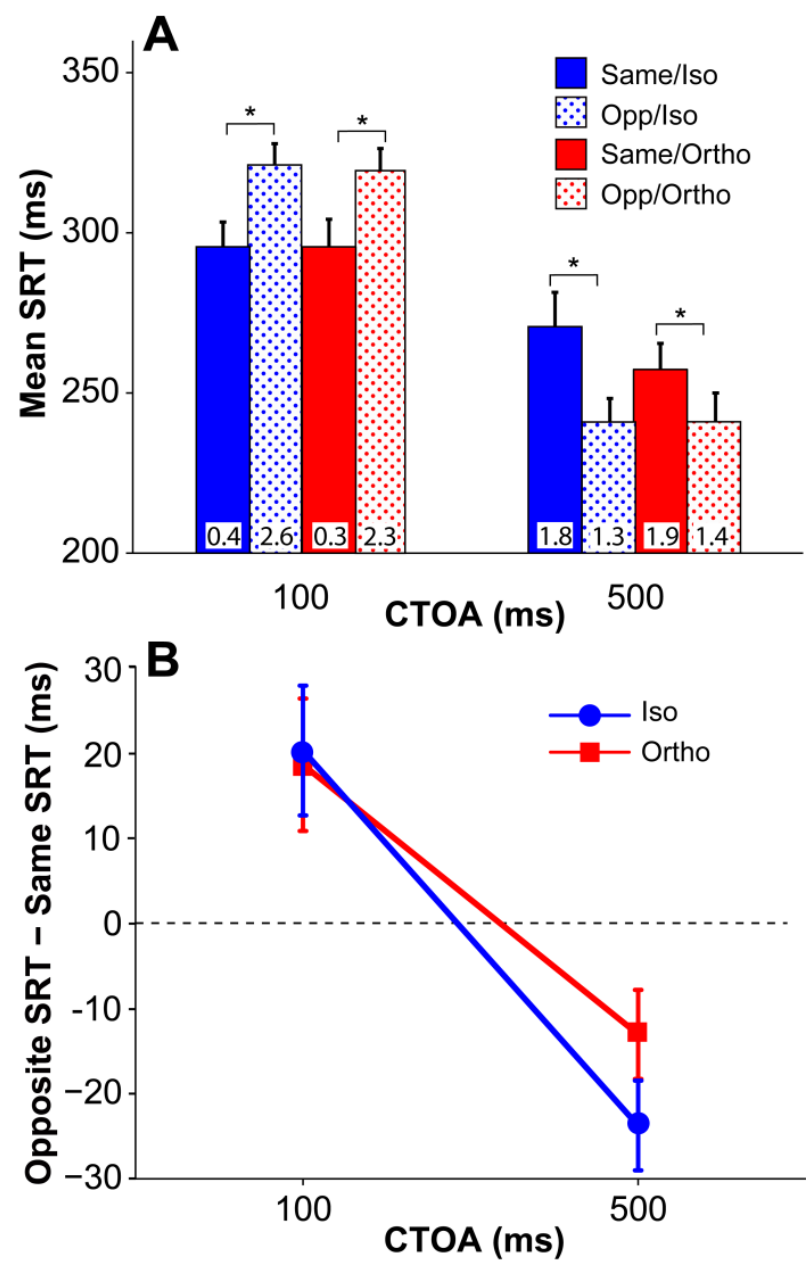

Figure 5: A. Mean SRT ( \pm 1 standard error) for the same (solid) versus opposite (stippled) cue-target locations across stimulus orientation conditions (Exp. 2): Cue and target were either the same (iso, blue) or orthogonal (ortho, red) orientation. Data were collected at CTOA of $100 \mathrm{~ms}$ and $500 \mathrm{~ms}$. The numbers within the bars indicate the percentage of the total saccade direction errors for a given condition. B. SRT differences for iso-oriented (blue) versus ortho-oriented (red) stimuli. ${ }^{*} p<0.05$, Bonferroni corrected. There was no significant effect of stimulus orientation.

\section{Discussion}

The goal of this study was to elucidate the mechanisms underlying the sensory based account of AC and IOR (Bell et al. 2004; Dorris et al. 2002; Fecteau et al. 2004; Fecteau and Munoz 2005). In Experiment 1 (Visual Occlusion Manipulation), we tested the hypothesis that $\mathrm{AC}$ and IOR rely on an early monocular stage of visual processing (i.e., the retina or LGN; Monocular Hypothesis), versus a later binocular stage of visual processing 
(Binocular Hypothesis; Fig. 1). The results of Experiment 1 clearly support the Binocular Hypothesis: AC and IOR were similar in monocular and interocular viewing conditions demonstrating that they must rely on processes that occur after the signals from both eyes converge, which excludes several monocular areas such as the retina and LGN (Fig. 1). However, this left several possibilities, which led to the hypothesis proposed in Experiment 2 (Stimulus Orientation Manipulation). Here, we explored further the Binocular Hypothesis by asking whether AC or IOR involve a pathway through orientation selective mechanisms in visual cortex, by exploiting orientation selectivity of early visual cortical pathways (Hubel and Wiesel 1968, 1975; Ferster \& Miller, 2000). The results supported the hypothesis that AC/IOR do not depend significantly on stimulus orientation.

There is some evidence that orientation information may be processed without V1 (Boyer et al. 2005), but V1 is certainly the dominant brain area where orientation tuning originates. Although we cannot entirely rule out the contribution of other pathways that might process orientation, by reducing target contrast to a peri-threshold level, only dominant orientation tuned mechanisms (e.g., orientation selective neurons in V1) should have played a significant role in shaping the results. Target contrast was precisely controlled at $3 \log$ units above each participant's detection threshold to prevent a target of one orientation from being detectible by mechanisms tuned to the orthogonal orientation. The results indicate that orientation selective mechanisms are unlikely to contribute to AC or IOR (Fig. 5). Taken together, these results suggest that $\mathrm{AC}$ and IOR arise from orientation-independent, binocular mechanisms (Fig. 1C).

Previous studies have implicitly suggested that modulation of activity in early monocular structures may contribute less to reflexive covert orienting than processing in binocular structures. For example, the initial finding that IOR is coded in environmental rather than retinal coordinates is consistent with a Binocular Hypothesis (Maylor and Hockey 1985; Posner and Cohen 1984). Studies measuring manual RTs (Tassinari and Berlucchi 1993), or event-related potentials (McDonald et al. 1999) presented stimuli interocularly in a cue-target task and both AC and IOR were found to transfer interocularly. However, these studies did not utilize monocular (same eye) presentation, so it was not clear that early monocular processing did not contribute to the manifestation of $\mathrm{AC}$ or IOR. A more recent study (Self \& Roelfsema, 2010) showed a significant monocular cueing effect with manual RTs at a short CTOA (50 ms) only when cue and target were presented to the same eye. However, this study may not be directly comparable to the current study because it used methods very different from traditional cueing studies, and required participants to maintain correct fusion of dichoptically presented stimuli using a mirror stereoscope. As such, SRTs may in fact look very different from manual RTs in this task given the eye strain that often accompanies the unnatural accommodation required for fusing dichoptic images. In addition, this study required participants to perform a discrimination task as part of the reaction time (i.e., participants had to manually respond whether the target was vertically or horizontally oriented). As noted earlier, comparisons with the binocular condition must be interpreted with some caution because excitation of the photoreceptors of the combined eyes would necessarily result in greater visual input into the brain than in the monocular or interocular condition (see for example the black bars and lines in Fig. 4). An unpublished doctoral thesis (Maylor 1983) did show approximately equivalent magnitude of IOR for monocular and interocular conditions, but it did not address the interocular transfer of AC, and did not include the interocular "opposite" condition, in which cue are target are presented at the opposite location, to different eyes. Our study extended these earlier findings in three important ways: 1) The experimental design included the full factorial combination of monocular, interocular, and binocular cue and target presentations which allowed direct comparisons across conditions; 2) We included a critical control task that ensured no leakage of visual signals during goggle occlusion; 3) We explored the Binocular Hypothesis by testing whether a pathway involving orientation selective mechanisms in visual cortex contributes to IOR or AC.

Our results suggest that visual cortical areas selective for orientation contribute little to AC or IOR. In a recent study by Patel et al. (2010), a cue and target of the same shape resulted in a smaller facilitation effect at the short CTOA, but no differences at the longer CTOA. Interestingly, Red et al. (2012) attributed this effect to a mechanism residing in the dorsal processing stream, which is not traditionally associated with shape selectivity. Similarly, Taylor and Donnelly (2002) reported that cue-target shape similarity had little effect on IOR provided it was task irrelevant. A similar result was reported by Pratt et 
al. (2001). Although it was not in the interest of these studies to use stimuli optimized for the visual tuning properties of neurons in early visual cortex (namely V1), our results are consistent with these earlier findings, in particular those associated with the longer CTOA. However, we also did not see any evidence of orientation dependency at the short CTOA (Fig 5B). Although this seems inconsistent with Patel et al. (2010), there are important differences in the stimuli between these studies, namely that we controlled stimulus visibility by determining within-subject detection thresholds, and our stimuli were optimized for V1 (Ferster and Miller 2000).

In a recent review, Sereno et al. (2010) reported that neurons in the lateral intraparietal area (LIP) show reduced responses to a repeated stimulus, and these findings have been used to suggest that this could be the upstream source of the attenuated signals observed in the $\mathrm{SC}$, or that the attenuation is an intrinsic property of neurons in several brain areas. As noted earlier there is strong evidence that IOR in particular must arise relatively early in the visual processing stream, because a neural correlate of it is already observed in the earliest part of the target related response in neurons from the SCs (Dorris et al. 2002; Fecteau and Munoz, 2005), which does not receive inputs from parietal or frontal cortices, but instead from the retina and V1 (White and Munoz, 2011). Furthermore, similar correlates of AC and IOR have been identified in several other visual areas (Constantinidis and Steinmetz 2001; Lepsien and Pollman 2002; Mayer et al. 2004; Prime and Ward 2006; Ro et al. 2003; Robinson et al. 1995; Sapir et al. 2004; Steinmetz et al. 1994). Thus, the neural mechanisms responsible for IOR are likely to emerge early in visual processing, and the effect may then be propagated to other brain areas (Fecteau et al. 2004; Fecteau and Munoz 2005; Fecteau and Munoz 2006). These observations limit to some degree the stage of visual processing that may contribute to IOR. The results of our study suggest that adaptation of the incoming sensory response (Boehnke \& Munoz, 2008; Boehnke et al. 2011) must emerge at one of the earliest stages of binocular visual processing (e.g, non-orientation channels through V1, input to the SCs, or the pretectum).

First, along the thalamocortical pathway, efferents from each eye are segregated into monocular layers in the LGN, and project to monocular simple cells in layer $4 \mathrm{c}$ of $\mathrm{V} 1$ that are segregated into eye-specific patches (Hubel and Wiesel 1968, 1975). These cells progressively syn- apse onto complex then hypercomplex cells in layers above and below 4c, and binocular convergence occurs later within V1 (Hubel and Wiesel 1968, 1975). Modulation of the target related response observed in IOR could occur as early as V1 binocular (non-orientation tuned) neurons, and then propagate to other visual areas, including the SCs and SCi (Fries, 1984; Lock et al., 2003). This hypothesis is supported by recent evidence that IOR (but not AC) is dramatically attenuated in monkeys with V1 lesion (Ikeda et al., 2011).

Second, the retina and visual cortex project directly to the SCs, where the majority of neurons respond equally well to stimuli presented monocularly or binocularly, and only a minority of cells respond to one eye only (Cynader and Berman 1972, Goldberg and Wurtz 1972, Hubel et al. 1975, Humphrey 1968, Schiller et al. 1974). Inputs to the SCs could represent a critical locus for IOR. The Dorris et al. (2002) study showed that the visual attenuation that is the neuronal signature of IOR must occur upstream of the SC neurons they recorded. Therefore, the processes that lead to IOR could occur at the synapse of the inputs to the SCs. Although signals invisible to the retinotectal path (S-cone signals) can still lead to robust AC and IOR (Sumner et al. 2002), corticotectal inputs could remain critical. Parvocellular-based (chromatic) singletons can also elicit oculomotor capture (Leonard and Luck, 2011), which is consistent with the fact that the SC is highly sensitive to both achromatic and chromatic signals, most likely arising from corticotectal inputs (White et al. 2009). The SCs is ideally situated to relay these signals back to most visual cortical areas via the inferior Pulvinar nucleus (Berman \& Wurtz, 2010; see also Boehnke and Munoz 2008). This would be consistent with many studies that have reported correlates of $\mathrm{AC}$ and IOR in several other visual areas (Constantinidis and Steinmetz 2001; Lepsien and Pollman 2002; Mayer et al. 2004; Prime and Ward 2006; Ro et al. 2003; Robinson et al. 1995; Sapir et al. 2004; Steinmetz et al. 1994). In a recent review of thalamic pathways for active vision, Wurtz et al. (2011) argued that these ascending SC-tocortex pathways may contribute to the neuronal mechanisms that underlie visuospatial attention. The observations described in our study are certainly consistent with this view.

Finally, the retina also projects directly to two subnuclei of the pretectum: the nucleus of the optic tract (NOT) and the pretectal olivary nucleus (PON) (Gamlin, 
2005). It is unknown whether the PON projects to the SC (Benevento et al. 1977; Edwards et al. 1979), or whether the SC-projecting NOT cells are the same cells which receive retinal input (Nunes Cardozo et al. 1994), but the pretectum does project to many other structures in the brain, including the thalamus, midbrain, pons, medulla, subthalamus, hypothalamus, and cortex (Gamlin, 2005). However, the pretectum is a less likely candidate of IOR, because it would have to receive, process, and resend this attenuated response to the rest of the brain before the SCs receives visual information directly from the retina and V1.

Although this study was motivated by the sensory account of $\mathrm{AC} / \mathrm{IOR}$, we realize that this mechanism may not entirely account for the many variants of AC/IOR observed in the literature (e.g., contingent capture (Folk et al., 1992); environmental-based accounts of IOR (Posner and Cohen 1984; Maylor \& Hockey 1985; Hilchey et al., 2012); object-based IOR (Tipper et al., 1991)). A more definitive conclusion about the precise locus of the sensory mechanisms responsible for reflexive covert orienting will require direct neuronal measurements of modulated visual signals in other brain areas associated with these effects. Using a process-of-elimination approach, and integrating behavioral results with neuroanatomical evidence, the results of this study suggest that early monocular areas and orientation selective visual cortex cannot adequately explain the sensory based account of AC/IOR. More importantly, the results point to structures like the input to the SCs and non-orientation selective regions of early visual cortex as potential loci for further investigation.

\section{References}

Bell. A.H., Fecteau. J.H., \& Munoz, D.P. (2004). Using auditory and visual stimuli to investigate the behavioral and neuronal consequences of reflexive covert orienting. Journal of Neurophysiology, 91(5), 21722184.

Benevento, L.A., Rezak, M., \& Santos-Anderson, R. (1977) An autoradiographic study of the projections of the pretectum in the rhesus monkey (Macaca mulatta): evidence for sensorimotor links to the thalamus and oculomotor nuclei. Brain Research 127(2), 197218.
Berman, R.A., \& Wurtz, R.H. (2010). Functional identification of a pulvinar path from superior colliculus to cortical area MT. Journal of Neuroscience, 30, 63426354.

Blake, R., \& Fox, R. (1973). The psychophysical inquiry into binocular summation. Perception \& Psychophysics, 14(1), 161-185.

Boehnke, S.E., Berg, D.J., Marino, R.M., Baldi, P.F., Itti, L., \& Munoz, D.P. (2011). Visual adaptation and novelty responses in the superior colliculus. European Journal of Neuroscience, 34(4), 766-779.

Boehnke, S.E., \& Munoz, D.P. (2008). On the importance of the transient visual response in the superior colliculus. Current Opinion in Neurobiology, 18, $1-8$.

Boyer, J.L., Harrison, S., \& Ro, T. (2005). Unconscious processing of orientation and color without primary visual cortex. Proceedings of the National Academy of Sciences of the United States of America, 102(46), 16875-16879.

Constantinidis, C., \& Steinmetz, M.A. (2001). Neuronal responses in area 7a to multiple stimulus displays: II. responses are suppressed at the cued location. Cerebral Cortex, 11(7), 592-597.

Correa, A., Lupiáñez, J., Milliken, B., \& Tudela, P. (2004). Endogenous temporal orienting of attention in detection and discrimination tasks. Perception \& Psychophysics, 66(2), 264-278.

Cynader, M., \& Berman, N. (1972). Receptive-field organization of monkey superior colliculus. Journal of Neurophysiology, 35(2), 187-201.

Danziger, S., Fendrich, R., \& Rafal, R. (1997). Inhibitory Tagging of Locations in the Blind Field of Hemianopic Patients. Consciousness \& Cognition, 6(2), 291307.

Dorris, M.C., Klein, R.M., Everling, S., \& Munoz, D.P. (2002). Contribution of the primate superior colliculus to inhibition of return. Journal of Cognitive Neuroscience, 14(8), 1256-1263.

Edwards, S.B., Ginsburgh, C.L., Henkel, C.K., \& Stein, B.E. (1979). Sources of subcortical projections to the superior colliculus in the cat. Journal of Comparative Neurology, 184(2), 309-329.

Fecteau, J.H., Bell, A.H., \& Munoz, D.P. (2004). Neural correlates of the automatic and goal-driven biases in 
Journal of Eye Movement Research

6(1):1, 1-14

orienting spatial attention. Journal of Neurophysiology, 92(3), 1728-1737.

Fecteau, J.H., \& Munoz, D.P. (2005). Correlates of capture of attention and inhibition of return across stages of visual processing. Journal of Cognitive Neuroscience, 17(11), 1714-1727.

Fecteau, J.H., \& Munoz, D.P. (2006). Salience, relevance, and firing: a priority map for target selection. Trends in Cognitive Sciences, 10(8), 382-390.

Fecteau, J.H., \& Munoz, D.P. (2007). Warning signals influence motor processing. Journal of Neurophysiology, 97, 1600-1609.

Ferster, D., \& Miller, K.D. (2000). Neural mechanisms of orientation selectivity in the visual cortex. Annual Review of Neuroscience, 23, 441-471.

Folk, C.L., Remington, R.W., \& Johnston, J.C. (1992). Involuntary covert orienting is contingent on attentional control settings. Journal of Experimental Psychology: Human Perception and Performance, 18, 1030-1044.

Fries, W. (1984). Cortical projections to the superior colliculus in the macaque monkey: a retrograde study using horseradish peroxidase. Journal of Comparative Neurology, 230(1), 55-76.

Gamlin, P.D. (2005). The pretectum: connections and oculomotor-related roles. Progress in Brain Research, 151, 379-405.

Godijn, R., \& Theeuwes, J. (2002). Oculomotor Capture and Inhibition of Return. Psychological Research, 66, 234-246.

Goldberg, M.E., \& Wurtz, R.H. (1972). Activity of superior colliculus in behaving monkey. I. Visual receptive fields of single neurons. Journal of Neurophysiology, 35(4), 542-559.

Hays, A.V., Richmond, B.J., \& Optican, L.M. (1982). A UNIX-based multiple process system for real-time data acquisition and control. WESCON Conference Proceedings, 2, 1-10.

Hilchey, M.D., Klein, R.M., Satel, J., \& Wang, Z. (2012). Oculomotor inhibition of return: How soon is it "recoded" into spatiotopic coordinates? Attention, Perception \& Psychophysics, 74, 1145-1153.

Hubel, D.H., LeVay, S., \& Wiesel, T.N. (1975). Mode of termination of retinotectal fibers in macaque monkey:
Higenell, V., White, B.J., Hwang, J.R., \& Munoz, D.P. (2013) Mechanisms underlying sensory-based AC/IOR

an autoradiographic study. Brain Research, 96(1), 2540 .

Hubel, D.H., \& Wiesel, T.N. (1977). Ferrier lecture: Functional architecture of macaque monkey visual cortex. Proceedings of the Royal Society of London B: Biological Sciences, 198, 1-59.

Hubel, D.H., \& Wiesel, T.N. (1968). Receptive fields and functional architecture of monkey striate cortex. Journal of Physiology, 195(1), 215-243.

Humphrey, N.K. (1968). Responses to visual stimuli of units in the superior colliculus of rats and monkeys. Experimental Neurology, 20(3), 312-340.

Ikeda, T., Yoshida, M., \& Isa, T. (2011). Lesion of primary visual cortex in monkey impairs the inhibitory but not the facilitatory cueing effect on saccade. Journal of Cognitive Neuroscience, 23, 1160-1169.

Jonides, J. (1981). Voluntary versus automatic control over the mind's eye's movement. In: J.B. Long \& A.D. Baddeley (Eds), Attention and Performance IX (pp187-203), Erlbaum, New Jersey.

Klein, R.M. \& MacInnes, W.J. (1999). Inhibition of return is a foraging facilitator in visual search. Psychological Science, 10, 346-352.

Klein, R.M. (2009). On the Control of Attention. Canadian Journal of Experimental Psychology, 63(3), 240252.

Klein, R.M. (2000). Inhibition of return. Trends in Cognitive Sciences, 4(4), 138-147.

Leonard, C.J., \& Luck, S.J. (2011). The role of magnocellular signals in oculomotor attentional capture. Journal of Vision, 11(13), 1-12.

Lepsien, J., \& Pollmann, S. (2002). Covert reorienting and inhibition of return: an event-related fMRI study. Journal of Cognitive Neuroscience, 14(2), 127-144.

Lock, T.M., Baizer, J.S., \& Bender, D.B. (2003). Distribution of corticotectal cells in the macaque. Experimental Brain Research, 151(4), 455-470.

Macmillan, N.A., \& Creelman, C.D. (1991). Detection theory: A user's guide. New York: Cambridge University Press.

Mayer, A.R., Seidenberg, M., Dorflinger, J.M., \& Rao, S.M. (2004). An event-related fMRI study of exogenous orienting: supporting evidence for the cortical 
Journal of Eye Movement Research

6(1):1, 1-14

basis of inhibition of return? Journal of Cognitive Neuroscience, 16(7), 1262-1271.

Maylor, E. (1983). Components of Orienting in Visual Space. Unpublished doctoral thesis, University of Durham.

Maylor, E. (1985). Facilitatory and Inhibitory Components of Orienting in Visual Space. In: M. Posner \& O. Marin (Eds) Attention and Performance XI. (pp. 189-203), Erlbaum, New Jersey.

Maylor, E.A., \& Hockey, R. (1985). Inhibitory component of externally controlled covert orienting in visual space. Journal of Experimental Psychology: Human Perception and Performance,11(6), 777-787.

McDonald, J.J., Ward, L.M., \& Kiehl, K.A. (1999). An event-related brain potential study of inhibition of return. Perception \& Psychophyisics, 61(7), 14111423.

Minucci, P.K., \& Connors, M.M. (1964). Reaction time under three viewing conditions: binocular, dominant eye, and nondominant eye. Journal of Experimental Psychology, 67, 268-275.

Munoz, D.P., Broughton, J.R., Goldring, J.E., \& Armstrong, I.T. (1998). Age-related performance of human subjects on saccadic eye movement tasks. Experimental Brain Research, 121, 391-400.

Niemi, P., \& Näätänen, R. (1981). Foreperiod and simple reaction time. Psychological Bulletin, 89, 133-162.

Nunes Cardozo, B., Mize, R.R., \& Van der Want, J.J. (1994). GABAergic and non-GABAergic neurons in the nucleus of the optic tract project to the superior colliculus: an ultrastructural retrograde tracer and immunocytochemical study in the rabbit. Journal of Comparative Neurology, 350(4), 646-656.

Paré, M., \& Munoz, D.P. (1996). Saccadic reaction time in the advanced preparation of oculomotor programs is primarily responsible for express saccade occurrence. Journal of Neurophysiology, 76, 3666-3681.

Patel, S.S., Peng, X., \& Sereno, A.B. (2010). Shape effects on reflexive spatial selective attention and a plausible neurophysiological model, Vision Research, 50(13), 1235-1248.

Poffenberger, A.T. (1912). Reaction time to retinal stimulation with special reference to the time lost in conduction through nerve centers. Archives of Psychology, 23, 1-73.
Higenell, V., White, B.J., Hwang, J.R., \& Munoz, D.P. (2013) Mechanisms underlying sensory-based AC/IOR

Posner, M.I., \& Cohen, Y. (1984). Components of visual orienting. In: H. Bouma \& D.G. Bowhuis (Eds), In: Attention and Performance X., (pp. 531-556), Erlbaum, New Jersey.

Posner, M.I., Rafal, R.D., Choate, L.S., \& Vaughan, J. (1985). Inhibition of Return: Neural Basis and Function. Cognitive Neuropsychology, 2(3), 221-228.

Pratt, J., Hillis, J., \& Gold, J.M. (2001). The effect of the physical characteristics of cues and targets on facilitation and inhibition. Psychonomic Bulletin \& Review, 8, 489-495.

Prime, D.J., \& Ward, L.M. (2006). Cortical expressions of inhibition of return. Brain Research, 1072(1), 161174.

Red, S.D., Patel, S.S., \& Sereno, A.B. (2012). Shape effects on reflexive spatial attention are driven by the dorsal stream, Vision Research, 55(15), 32-40.

Ro, T., Farnè, A., \& Chang, E. (2003). Inhibition of return and the human frontal eye fields. Experimental Brain Research, 150, 290-296.

Robinson, D.L., Bowman, E.M., \& Kertzman, C. (1995). Covert orienting of attention in macaques. II. Contributions of parietal cortex. Journal of Neurophysiology, 74(2), 698-712.

Sapir, A., Hayes, A., Henik, A., Danziger, S., \& Rafal, R. (2004). Parietal lobe lesions disrupt saccadic remapping of inhibitory location tagging. Journal of Cognitive Neuroscience, 16(4), 503-509.

Sapir, A., Rafal, R., \& Henik, A. (2002). Attending to the thalamus: inhibition of return and nasal-temporal asymmetry in the pulvinar. Neuroreport, 13(5), 693697.

Sapir, A., Soroker, N., Berger, A., \& Henik, A. (1999). Inhibition of return in spatial attention: direct evidence for collicular generation. Nature Neuroscience, 2(12), 1053-1054.

Schiller, P.H., Stryker, M., Cynader, M., \& Berman, N. (1974). Response characteristics of single cells in the monkey superior colliculus following ablation or cooling of visual cortex. Journal of Neurophysiology, 37(1), 181-194.

Self, M.W., \& Roelfsema, P.R. (2010). A monocular, unconscious form of visual attention. Journal of $\mathrm{Vi}$ sion, 10(4), 1-22. 
Sereno, A.B., Briand, K.A., Amador, S.C., \& Szapiel, S. (2006). Disruption of reflexive attention and eye movements in an individual with a collicular lesion. Journal of Clinical \& Experimental Neuropsychology, 28(1), 145-166.

Sereno, A.B., Lehky, S.R., Patel, S.S., \& Peng, X. (2010). A neurophysiological correlate and model of reflexive spatial attention. In N. Srinivasan, B. Kar, \& J. Pandey (Eds.), Advances in cognitive science. (pp. 104132). New Delhi: SAGE Publications India Pvt Ltd.

Shulman, G.L. (1984). An asymmetry in the control of eye movements and shifts of attention. Acta Psychologica, 55(1), 53-69.

Steinmetz, M.A., Connor, C.E., Constantinidis, C. \& McLaughlin, J.R. (1994). Covert attention suppresses neuronal responses in area $7 \mathrm{a}$ of the posterior parietal cortex. Journal of Neurophysiology, 72(2), 10201023 .

Sumner, P., Adamjee, T., \& Mollon, J.D. (2002). Signals invisible to the collicular and magnocellular pathways can capture visual attention. Current Biology, 12, 1312-1316.

Taylor, T.L., \& Donnelly, M.P.W. (2002). Inhibition of return for target discriminations: The effect of repeating discriminated and irrelevant stimulus dimensions. Perception \& Psychophysics, 64, 292-317.

Tassinari, G., \& Berlucchi, G. (1993). Sensory and attentional components of slowing of manual reaction time to non-fixated visual targets by ipsilateral primes. $\mathrm{Vi}$ sion Research, 33(11), 1525-1534.

Tipper, S.P., Driver, J., \& Weaver, B. (1991). Objectcentered inhibition of return of visual attention. The Quarterly Journal of Experimental Psychology A, 43, 289-298.

Tipper, C., \& Kingstone, A. (2005). Is inhibition of return a reflexive effect?, Cognition, 97, B55-B62.

White, B.J., Boehnke, S.E., Marino, R.A., Itti, L., \& Munoz, D.P. (2009). Color-related signals in the primate superior colliculus. Journal of Neuroscience, 29, 12159-12166.

White, B.J., \& Munoz, D.P. (2011). The superior colliculus. In S. Liversedge, I. Gilchrist, \& S. Everling (Eds.), Oxford handbook of eye movements (1st ed.), (pp. 195-213), New York: Oxford University Press.
Wurtz, R.H., McAlonan, K., Cavanaugh, J., \& Berman, R.A. (2011). Thalamic pathways for active vision. Trends in Cognitive Sciences, 15(4), 177-184. 